\title{
Article \\ Overexpression of the Apple (Malus $\times$ domestica) MdERF100 in Arabidopsis Increases Resistance to Powdery Mildew
}

\author{
Yiping Zhang 1,2,+ (D) Li Zhang 1,2,+ , Hai Ma 1,2, Yichu Zhang 1,2, Xiuming Zhang 1,2, Miaomiao Ji 1,2, \\ Steve van Nocker ${ }^{3}$, Bilal Ahmad ${ }^{1,2} \mathbb{D}$, Zhengyang Zhao ${ }^{1,2}$, Xiping Wang 1,2,*价 and Hua Gao ${ }^{1,2, *}$ \\ 1 State Key Laboratory of Crop Stress Biology in Arid Areas, College of Horticulture, \\ Northwest A\&F University, Xianyang 712100, China; zyp9709@nwafu.edu.cn (Y.Z.); \\ zhangli0221@nwafu.edu.cn (L.Z.); mahai@nwafu.edu.cn (H.M.); jyl74@nwsuaf.edu.cn (Y.Z.); \\ zhangxiuming@nwafu.edu.cn (X.Z.); jimiaomiao@nwafu.edu.cn (M.J.); bajwa1999@nwafu.edu.cn (B.A.); \\ zhaozy@nwsuaf.edu.cn (Z.Z.) \\ 2 Key Laboratory of Horticultural Plant Biology and Germplasm Innovation in Northwest China, \\ Ministry of Agriculture, Northwest A\&F University, Xianyang 712100, China \\ 3 Department of Horticulture, Michigan State University, East Lansing, MI 48824, USA; vannocke@msu.edu \\ * Correspondence: wangxiping@nwsuaf.edu.cn (X.W.); gaohua@nwafu.edu.cn (H.G.); \\ Tel.: +86-29-87082129 (X.W.); +86-29-87082613 (H.G.) \\ + These authors have equally contributed to this work.
}

check for updates

Citation: Zhang, Y.; Zhang, L.; Ma, H.; Zhang, Y.; Zhang, X.; Ji, M.; van Nocker, S.; Ahmad, B.; Zhao, Z.; Wang, X.; et al. Overexpression of the Apple (Malus $\times$ domestica) MdERF100 in Arabidopsis Increases Resistance to Powdery Mildew. Int. J. Mol. Sci. 2021, 22, 5713. https://doi.org/ $10.3390 /$ ijms 22115713

Academic Editor: Maria R. Ercolano

Received: 21 April 2021

Accepted: 19 May 2021

Published: 27 May 2021

Publisher's Note: MDPI stays neutral with regard to jurisdictional claims in published maps and institutional affiliations.

Copyright: (c) 2021 by the authors. Licensee MDPI, Basel, Switzerland. This article is an open access article distributed under the terms and conditions of the Creative Commons Attribution (CC BY) license (https:// creativecommons.org/licenses/by/ $4.0 /)$.

\begin{abstract}
APETALA2/ETHYLENE RESPONSIVE FACTOR (AP2/ERF) transcription factors play important roles in plant development and stress response. Although AP2/ERF genes have been extensively investigated in model plants such as Arabidopsis thaliana, little is known about their role in biotic stress response in perennial fruit tree crops such as apple (Malus $\times$ domestica). Here, we investigated the role of MdERF100 in powdery mildew resistance in apple. MdERF100 localized to the nucleus but showed no transcriptional activation activity. The heterologous expression of MdERF100 in Arabidopsis not only enhanced powdery mildew resistance but also increased reactive oxygen species (ROS) accumulation and cell death. Furthermore, MdERF100-overexpressing Arabidopsis plants exhibited differential expressions of genes involved in jasmonic acid (JA) and salicylic acid (SA) signaling when infected with the powdery mildew pathogen. Additionally, yeast two-hybrid and bimolecular fluorescence complementation assays confirmed that MdERF100 physically interacts with the basic helix-loop-helix (bHLH) protein MdbHLH92. These results suggest that MdERF100 mediates powdery mildew resistance by regulating the JA and SA signaling pathways, and MdbHLH92 is involved in plant defense against powdery mildew. Overall, this study enhances our understanding of the role of $M d E R F$ genes in disease resistance, and provides novel insights into the molecular mechanisms of powdery mildew resistance in apple.
\end{abstract}

Keywords: apple; MdERF100; MdbHLH92; powdery mildew; disease resistance

\section{Introduction}

Apple (Malus $\times$ domestica) is one of the most widely cultivated and economically valuable fruit crops worldwide [1]. In China, improvements in apple production technology have led to its cultivation in new areas. However, apple production is constantly challenged by biotic and abiotic pressures. Apple powdery mildew, caused by the obligate vegetative fungus Podosphaera leucotricha, is one of the most ubiquitous and devastating diseases in apple worldwide [2]. This fungus damages various tissues and organs of apple trees, including new buds, leaves, flowers and young fruits. Powdery mildew symptoms first appear on the leaves in the form of leaf curling, appearance of white powder on the leaf blade, drying of leaf tips and leaf abscission [3]. Apple producers commonly use fungicides to reduce the losses caused by powdery mildew, but the continuous use of fungicides has negative impacts on fruit production and the environment [4]. Therefore, it is essential to 
characterize the molecular basis of powdery mildew resistance and to develop powdery mildew-resistant cultivars.

The APETALA2/ETHYLENE RESPONSE FACTOR (AP2/ERF) genes encode plantspecific transcription factors, which perform important functions in plant development and response to various stresses, including pathogen infection, salt, wounding, drought, hypoxia and high temperatures $[5,6]$. The ERF genes form the largest subfamily of the $A P 2 / E R F$ gene family and contain an AP2 domain [7]. ERF genes have been well studied in the model plant Arabidopsis thaliana in the context of resistance to various pathogens, including Ralstonia solanacearum, Alternaria alternata, tobacco mosaic virus (TMV), Botyosphaeria dothidea, and Pseudomonas syringae pv. tomato DC3000 (PstDC3000). These genes have also been studies in other plant species. For example, heterologous expression of the cotton (Gossypium hirsutum) ERF gene GhB301 in Nicotiana benthamiana enhanced its resistance against fusarium wilt [8]. The basic helix-loop-helix (bHLH) superfamily is considered as the second largest class of plant transcription factors [9]. A bHLH domain contains 50-60 amino acids and two functionally distinct regions: the N-terminal basic region, which is involved in the binding to the E-box (CANNTG) DNA sequence, and the HLH domain, which mediates domain dimerization $[10,11]$. Numerous investigations have shown that bHLH transcription factors play important roles in abiotic stresses, including cold [12], drought [13], salinity [14] and iron deficiency [15]. Moreover, it has been shown that bHLHs participate in plant defense against pests and pathogens, such as Xanthomonas euvesicatoria [16]. Some pathogensis-related $(P R)$ genes, involved in salicylic acid (SA) signaling, were downregulated in the OsHLH61 RNA interference (RNAi) plants [17], indicating a close relationship between bHLHs and the SA signaling pathway.

Plant hormones such as SA and jasmonic acid (JA) play crucial roles in various defensive responses [18]. In Arabidopsis, the ENHANCED DISEASE SUSCEPTIBILITY 1 (EDS1) gene encodes a lipase-like nucleo-cytoplasmic protein that regulates SA accumulation $[19,20]$. The NONEXPRESSOR OF PR1 (NPR1) gene encodes an SA receptor, which is critical for SA-mediated defense responses and pathogen resistance [19,21]. In Arabidopsis, PR proteins encoded by AtPR2, AtPR3 and AtPR5 genes are involved in SA and JA regulation [22-24]. The defense gene AtPDF1.2 also plays an important role in JA-mediated defense pathways [25].

Thus far, our understanding of the defense molecular mechanisms of powdery mildew is limited. In grapevine, the expression of the VqSTS6 gene from Vitis quinquangularis enhanced powdery mildew resistance by increasing the content of stilbenes [8]. The ectopic expression of $V v D O F 3$ in Arabidopsis conferred enhanced resistance to powdery mildew through the SA signaling pathway [26]. The overexpressing of ERF1-V in wheat (Triticum aestivum) could improve resistance to powdery mildew [27]. Meanwhile, only a limited number of studies have addressed the molecular mechanisms of powdery mildew susceptibility and resistance in apple. The knockdown of the susceptibility $(S)$ gene MdMLO19 in apple resulted in enhanced resistance against powdery mildew [4]. Similarly, transgenic apple plants expressing the MhNPR1 gene from Malus hupehensis also showed enhanced resistance against powdery mildew [28]. Furthermore, there have been no reports on the potential role of ERF genes in powdery mildew resistance in apple. According to the previous study, MdERF100 was a differentially expressed gene selected from the transcriptome data of apple inoculated with Podosphaera leucotricha [1]. We suspect that MdERF100 functions as a positive regulator in powdery mildew resistance. To confirm our hypothesis, we performed a heterologous expression analysis of MdERF100 in Arabidopsis. In a futher study, we showed that MdERF100 physically interacts with MdbHLH92, and plays a positive role in SA-mediated defense against powdery mildew. The research aims to provide candidate genes for the development of powdery mildew-resistant apple cultivars, and lay the foundation for the investigation of the molecular mechanisms of disease resistance in apple. 


\section{Results}

\subsection{Expression Pattern and Characterization of MdERF100}

To evaluate the potential role of MdERF100 in disease resistance in apple, we analyzed its expression in the leaves of apple seedlings inoculated with Podosphaera leucotricha. The results showed that the expression of MdERF100 was strongly upregulated at $6 \mathrm{~h}$ post-inoculation (hpi), maintained at least until $12 \mathrm{hpi}$, downregulated at 24 and $48 \mathrm{hpi}$ and significantly upregulated at 72 hpi (Figure 1a). To determine whether MdERF100 responds to hormone signaling pathways, we treated apple leaves with SA, JA and ethephon (Eth), and monitored MdERF100 expression by quantitative real-time PCR (qRT-PCR). Treatment with all three hormones significantly upregulated the expression of MdERF100 (Figure 1b-d). Compared with the control, the expression of MdERF100 was up to 25.1-fold higher in the SA treatment at 12 hpi (Figure 1b) and 8.6- and 3.9-fold higher in the JA and Eth treatments, respectively, at 3 hpi (Figure 1c,d). The results suggest that MdERF100 functions as an important regulator in apple powdery mildew defense via the SA, JA, and Eth signaling pathways.

The MdERF100 gene is located on chromosome 06 on the apple genome (Apple Genome Browser: https:/ / www.rosaceae.org, accessed on 10 March 2020). The coding sequence (CDS) of MdERF100 is $831 \mathrm{bp}$ in length and is predicted to encode a 276-amino acid (aa) protein, with a molecular weight of $\sim 29.6 \mathrm{kDa}$ and an isoelectric point (pI) of 8.45 (Figure 1e). The MdERF100 protein contains a highly conserved AP2 domain (142-206 aa), as shown by the multiple sequence alignment of ERF proteins (Figure 1f). Additionally, phylogenetic analysis showed that MdERF100 is most closely related to PbERF2-like and PpERF1A (Figure 1g).

\subsection{MdERF100 Shows Nuclear Localization but No Transcriptional Activation Activity}

To determine the subcellular location of MdERF100, the open reading frame (ORF) of MdERF100 was subcloned downstream of the cauliflower mosaic virus (CaMV) 35S promoter with the green fluorescent protein (GFP), and the resulting 35S:MdERF100-GFP was transiently transformed into Nicotiana benthamiana leaf epidermal cells (Figure 2a). Leaf sections around the injection site were analyzed by confocal microscopy at approximately $24 \mathrm{~h}$ post-infiltration. The green fluorescence signal was constrained to several discrete foci corresponding to the position of nuclei, revealing that the MdERF100-GFP fusion localized to the nucleus (Figure 2a). To determine the transcriptional activation potential of MdERF100, we performed yeast two-hybrid (Y2H) assays. All yeast cells grew well on synthetic-defined medium lacking tryptophan (SD/-Trp). Yeast cells transformed with pGBKT7-MdERF100, empty vector (pGBKT7) or negative control (co-transformation of pGBKT7-Lam with pGADT7-T) did not survive on the SD medium lacking Trp, $X$ $\alpha$-Gal and Aureobasidin A (SD/-Trp/X- $\alpha-\mathrm{Gal} / \mathrm{AbA})$, while those transformed with the positive control (co-transformation of pGBKT7-53 with pGADT7-T) produced blue colonies, indicating that MdERF100 exhibited no activation ability in yeast (Figure 2b).

\subsection{Heterologous Expression of MdERF100 in Arabidopsis Confers Powdery Mildew Resistance}

To determine whether MdERF100 promotes disease resistance when expressed heterologously in Arabidopsis, we engineered transgenic Arabidopsis lines expressing MdERF100 under the control of the strong, constitutive CaMV 35S promoter (35S:MdERF100), and evaluated their resistance to powdery mildew. Three transgenic lines significantly overexpressing MdERF100 compared with wild-type (WT) were selected for further experiments (Figure S1). The 35S:MdERF100 transgenic lines showed enhanced resistance to powdery mildew compared with WT control plants (Figure 3a). All three 35S:MdERF100 transgenic lines showed significantly fewer and less severe symptoms, as indicated by the disease severity index. In addition, significantly fewer spores were recovered from the leaves of transgenic lines than from those of WT (Figure 3b). Furthermore, we stained the leaves of transgenic and WT plants with trypan blue, diaminobenzidine (DAB) and nitroblue tetrazolium (NBT) to visualize dead cells and the accumulation of reactive oxygen species 
(ROS) (Figure 3c). The results showed that the number of dead cells and the level of ROS were higher in transgenic lines than in the WT controls. These results indicate that powdery mildew resistance is accompanied by a strong allergic and lethal reaction because of ROS burst after invasion by the fungal pathogen.

(a)

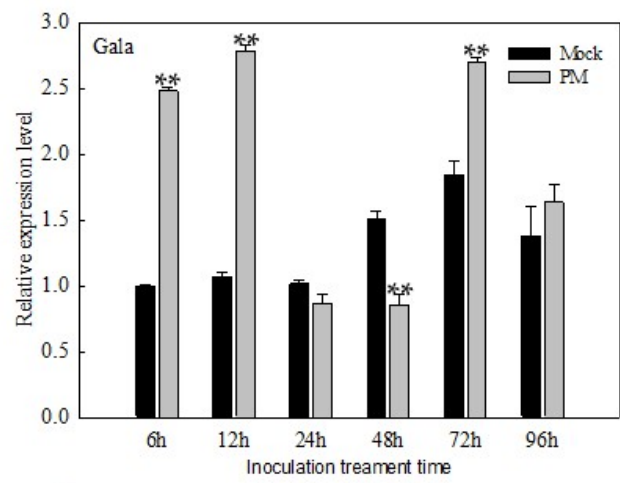

(c)

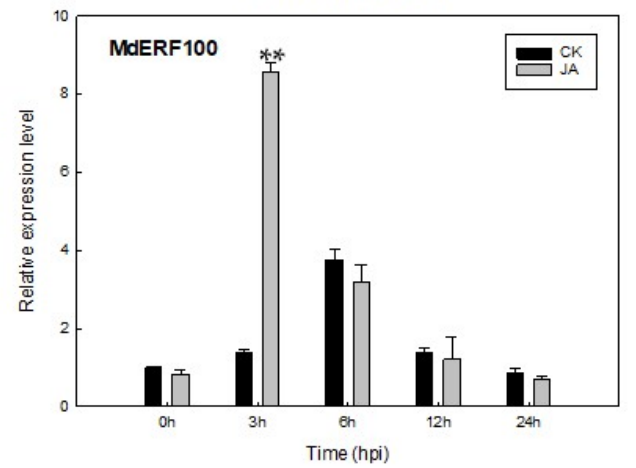

(e)

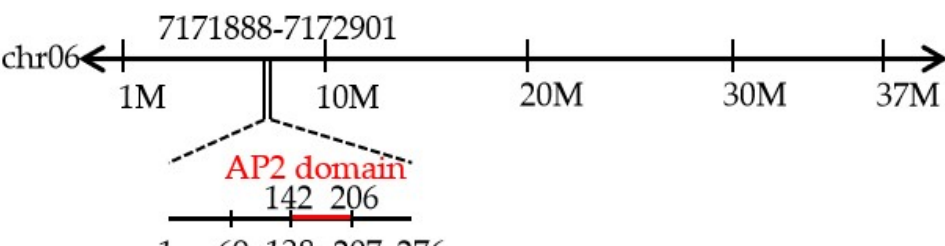

(g)

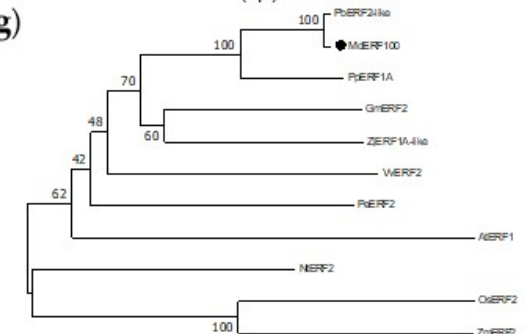

$1 \quad 69138207276$ (d)
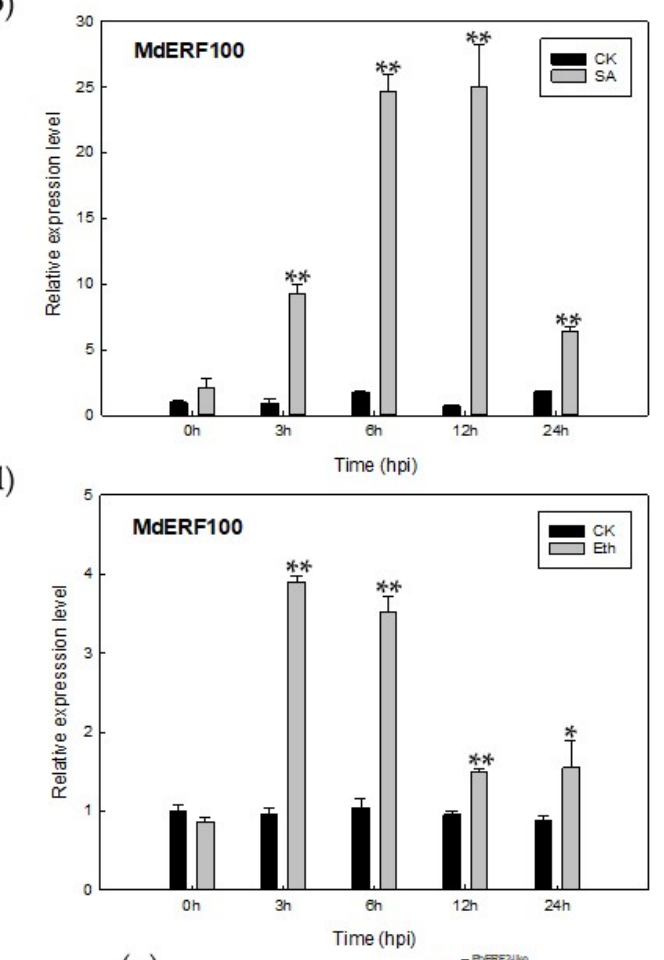

(b)

(f)

MAERE 100
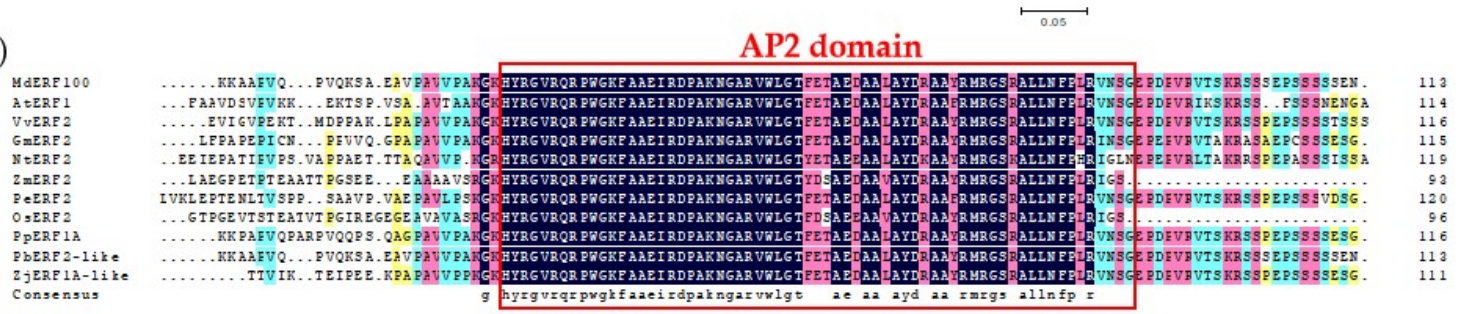

Figure 1. Expression and sequence analysis of MdERF100 isolated from the apple cultivar Gala. (a) Quantitative real-time PCR (qRT-PCR) analysis of MdERF100 in plants infected with powdery mildew. The x-axis indicates the time of sampling after inoculation, and the y-axis shows the relative transcription level. Error bars were calculated from three biological experiments, and show the standard deviation of the mean. Asterisks indicate statistical significance ${ }^{*} p<0.05,{ }^{* *} p<0.01$; Student's t-test). (b-d) Expression profile of MdERF100 in leaves treated with salicylic acid (SA) (b), jasmonic acid (JA) (c) and ethephon (Eth) (d,e) Chromosomal location of MdERF100. (f) Multiple sequence alignment of MdERF100 and its homologs in 10 different species. The conserved ERF domain is outlined in red. The accession numbers of the ERFs are as follows: PbERF2-like (XP009369170.1), PpERF1A (XP007209449.1), GmERF2 (XP003538752.2), ZjERF1A-like (XP015896697.1), VvERF2 (RVW50777.1), PeERF2 (XP011031947.1), AtERF1 (NP567530.4), NtERF2 (NP001311965.1), OsERF2 (XP015623259.1), ZmERF2 (PWZ22577.1). (g) Phylogenetic analysis of MdERF100 and its homologs. 
(a)

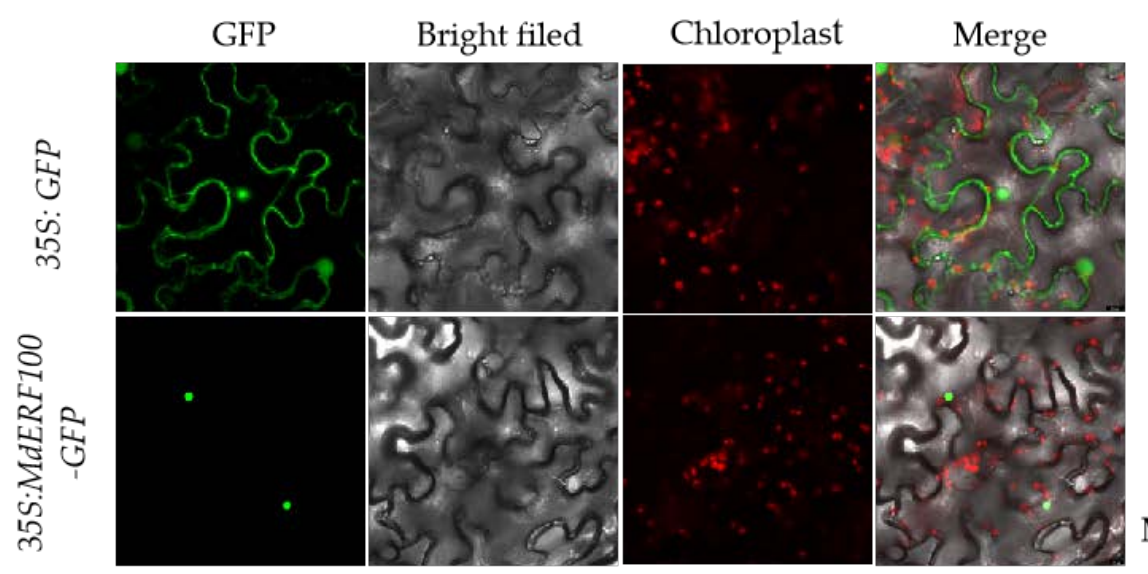

(b)

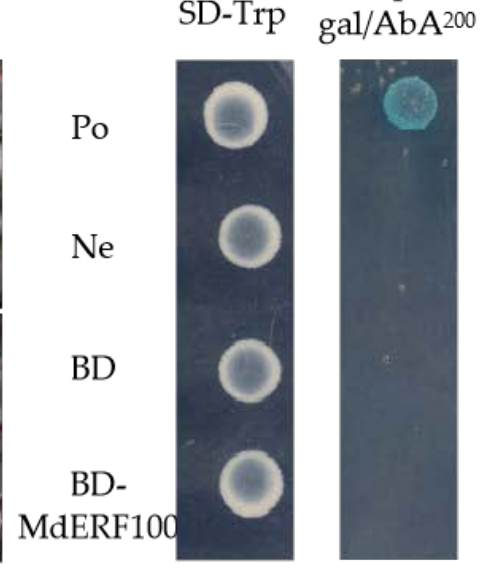

Figure 2. Subcellular localization and transactivation analysis of MdERF100. (a) Subcellular localization of MdERF100 in Nicotiana benthamiana plants. The pCAMBIA2300-GFP empty vector was used as control. Scale bar $=10 \mu \mathrm{m}$. (b) Transcriptional activation analysis of MdERF100 in yeast. Co-transformation of pGBKT7-53 with pGADT7-T and that of pGBKT7-Lam with pGADT7-T into yeast cells were used as positive and negative controls, respectively.

(a)

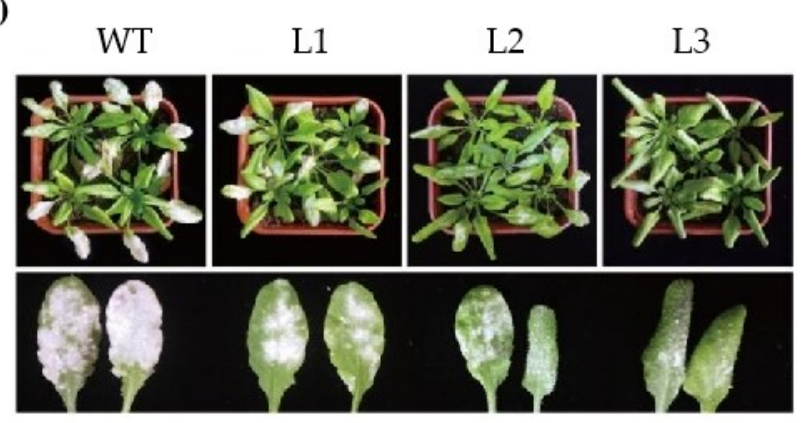

(b)

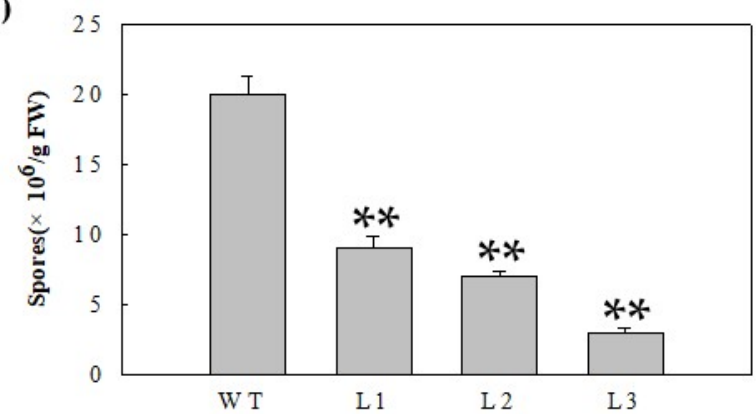

(c)

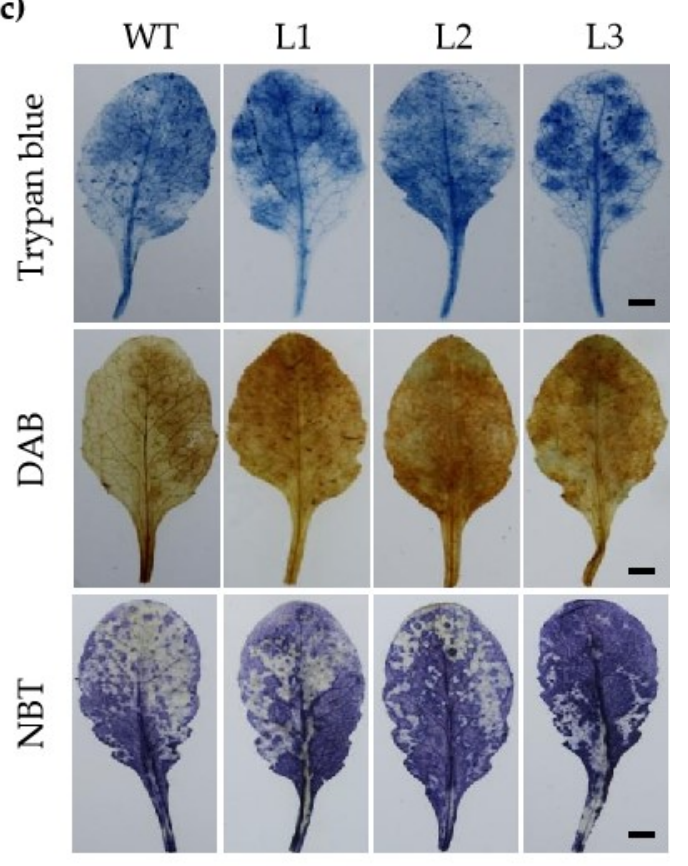

Figure 3. Response of MdERF100-overexpressing Arabidopsis lines to powdery mildew infection. (a) Phenotypes of wild-type (WT) plants and transgenic lines (L1, L2, L3) infected with powdery mildew. (b) Number of spores per gram of fresh leaves at $7 \mathrm{dpi}$ (days post-inoculation). Error bars show the standard deviation of the mean. Asterisks indicate statistically significant differences $(* * p<0.01$; Student's $t$-test). Experiments were repeated three times with consistent results. (c) Cell death and $\mathrm{O}^{2-}$ accumulation after pathogen inoculation. Scale bar $=10 \mathrm{~mm}$.

2.4. Expression Analysis of Disease Resistance (R) Genes in MdERF100-Overexpressing Plants in Response to E. necator Inoculation

To understand the mechanism of enhanced powdery mildew resistance in MdERF100overexpressing plants, we analyzed the relative expression levels of key genes involved in hormone biosynthesis or signal transduction pathways. These included genes involved in SA signaling (AtEDS1, AtPR2, AtPR5 and AtNPR1) and JA signaling (AtPDF1.2 and AtPR3) (Figure 4). The expression of these genes was examined by quantitative PCR (qPCR) 
in 4-week-old seedlings of all three 35S:MdERF100 transgenic lines at 0, 24, 72 and 120 hpi. The expression of AtEDS1 was higher at 24 and 72 hpi compared with the inoculated control (WT) plants, and was less than those in the control plants by 120 hpi (Figure 4a). Both AtNPR1 and AtPR2 were significantly upregulated at 24, 72 and $120 \mathrm{hpi}$ in all three transgenic lines (Figure $4 \mathrm{~b}, \mathrm{c}$ ). In addition, AtPR5 showed a significantly greater expression at 24 and $72 \mathrm{hpi}$ in all three transgenic lines, and relatively less expression at $120 \mathrm{hpi}$ in two transgenic lines (Figure 4f). However, both AtPDF1.2 and AtPR3 were downregulated at 24, 72 and $120 \mathrm{hpi}$ in transgenic lines compared with WT plants (Figure 4d,e). These results suggest that MdERF100 positively regulates resistance to powdery mildew via the SA signaling pathway but negatively regulates powdery mildew resistance through the JA signaling pathway.

(a)

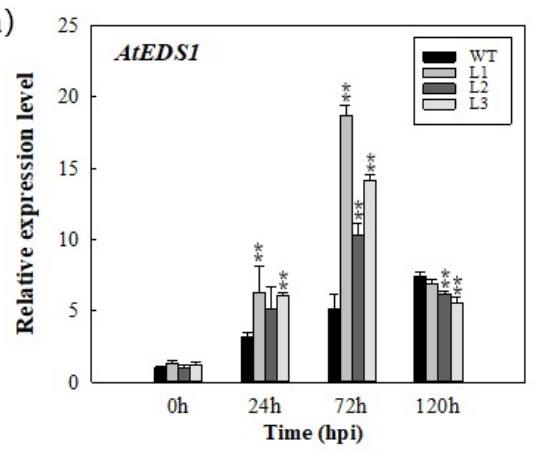

(d)

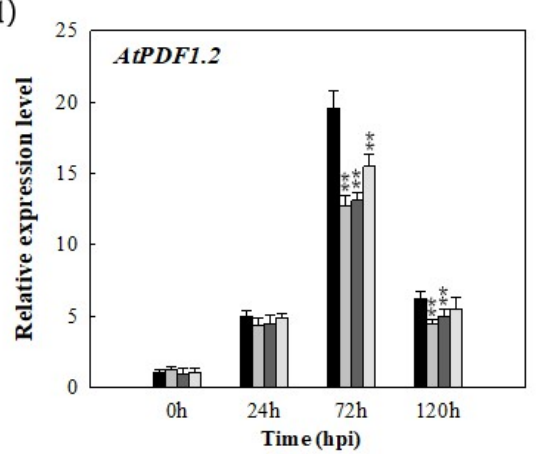

(b)

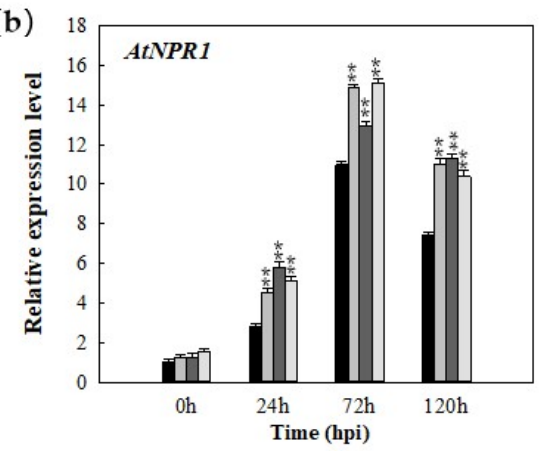

(e)

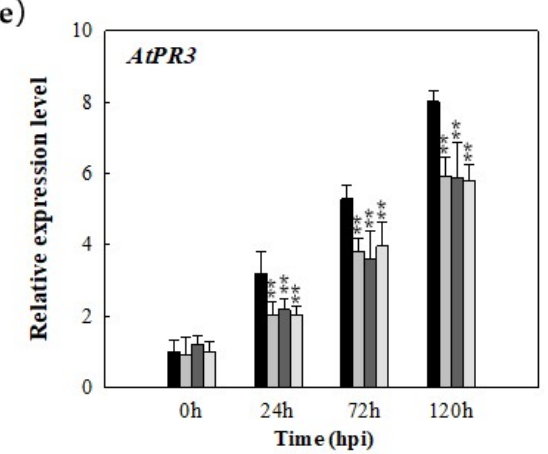

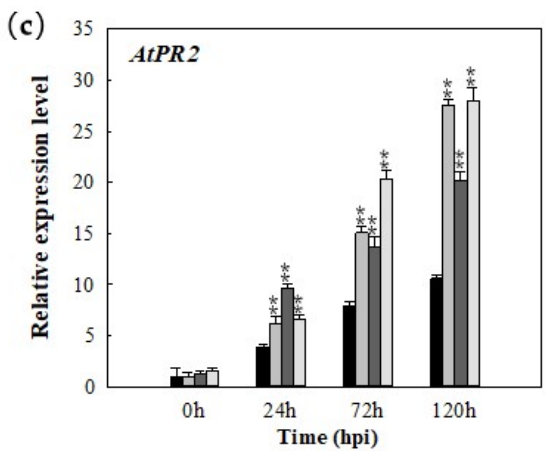

(f)

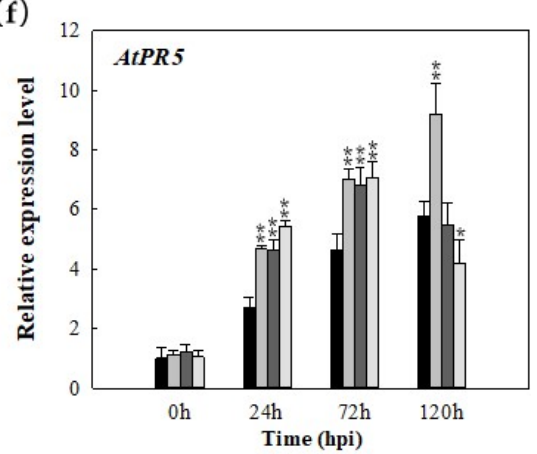

Figure 4. Expression analysis of disease resistance genes in transgenic Arabidopsis. (a-f) Expression profiles of AtEDS1 (a), AtNPR1 (b), AtPR2 (c), AtPDF1.2 (d), AtPR3 (e) and AtPR5 (f) at 0, 24, 72 and 120 hpi (hours post-inoculation). Error bars were calculated from three biological experiments, and show the standard deviation of the mean. Asterisks indicate statistical significance ${ }^{*} p<0.05,{ }^{* *} p<0.01$; Student's $t$-test).

\subsection{MdERF100 Physically Interacts with MdbHLH92}

We used the $\mathrm{Y} 2 \mathrm{H}$ system to test for interactions between MdERF100 and five putative interacting partners, including MdCSC1, MdBIP, MdbHLH92, MdPAE1 and MdUBQ, which were obtained from the STRING database, and genes encoding these proteins were cloned from the apple cultivar Gala. MdERF100 was fused to the DNA-binding domain (BD) in the pGBKT7 (Clonetech, Palo Alto, CA, USA) vector, while genes encoding the putative interacting partners were fused to the activation domain (AD) in the pGADT7 (Clonetech, Palo Alto, CA, USA) vector. The MdERF100 construct was co-transformed with each of the AD constructs into yeast cells. The co-transformed yeast cells cultured on SD/-Trp/-Leu (double dropout (DDO) medium) produced white colonies, whereas only yeast cells co-transformed with MdbHLH92 and cultured on SD/-Ade-His-Leu-Trp (quadruple dropout (QDO) medium) with $\mathrm{X}-\alpha-\mathrm{Gal}$ and $\mathrm{AbA}$ produced blue colonies (Figure 5a). Moreover, as shown in Figure $5 b$, yellow fluorescent signals were observed in protoplasts co-expressing MdERF100 and MdbHLH92. However, no fluorescent signal was detected in protoplasts transformed with other plasmid combinations (Figure $5 b$ ), 
indicating that MdERF100 interacted specifically with MdbHLH92 in yeast cells and Arabidopsis protoplasts (Figure 5).

(a)

BD-MdERF100

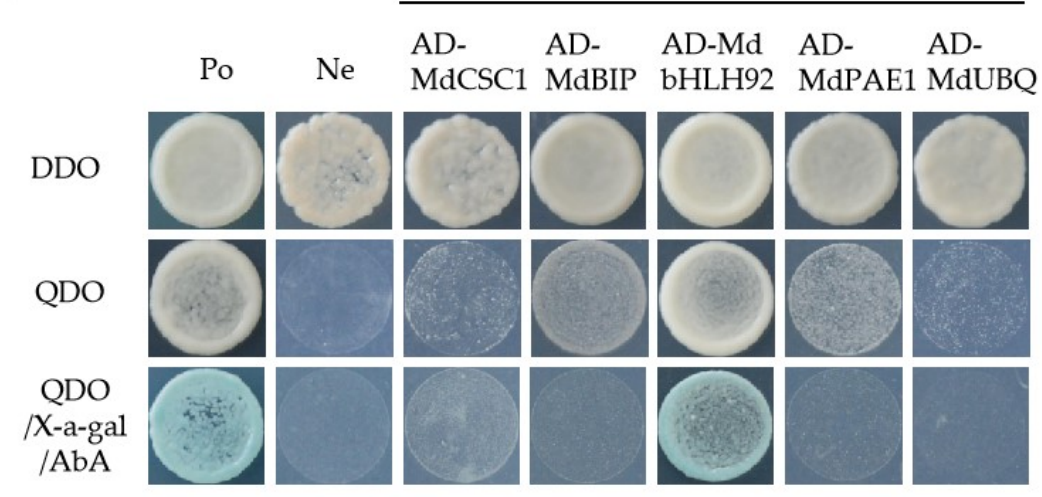

(b)

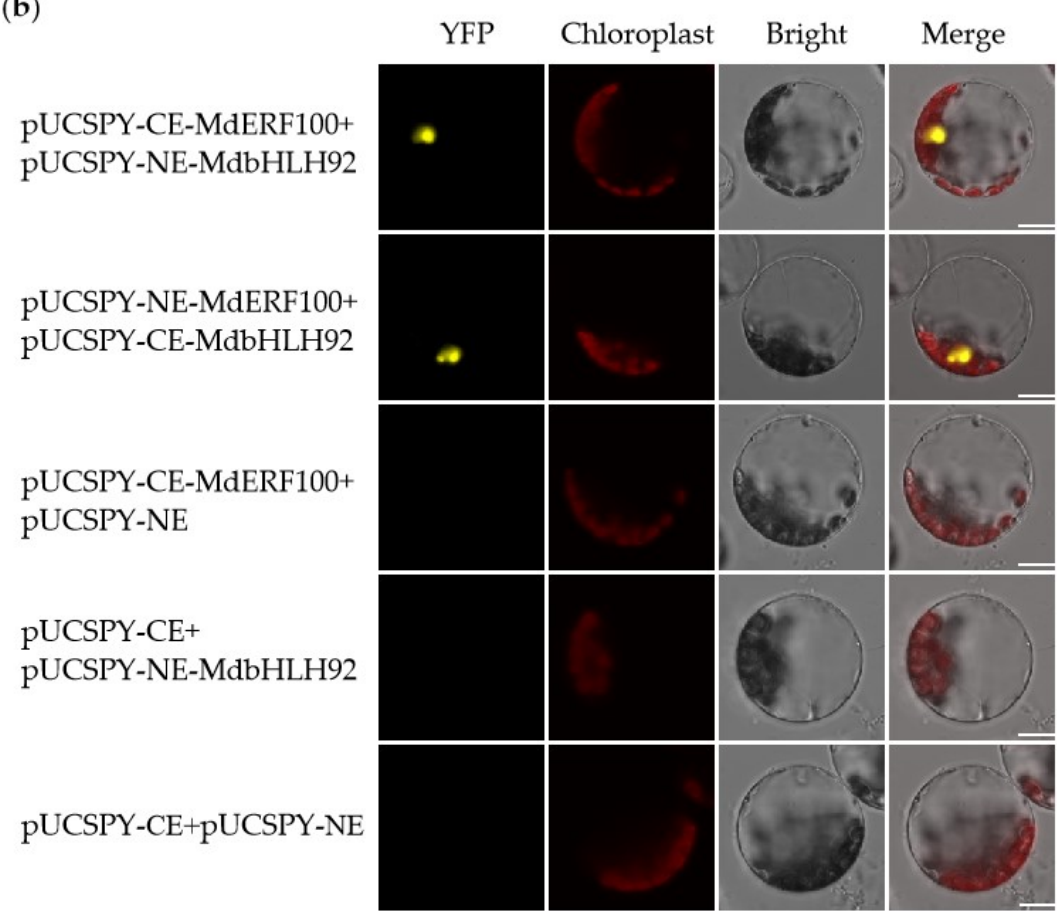

Figure 5. Verification of interaction between MdERF100 and MdbHLH92. (a) Yeast two-hybrid (Y2H) assay. The accession numbers of putative interacting partners of MdERF100 are as follows: MdCSC1 (XP_008373502.2), MdBIP (XP_008390320.1), MdPAE1 (XP_008371892.1), MdUBQ (XP_008367744.1). Yeast co-transformed with pGBKT7-53 and pGADT7-T or with pGBKT7-Lam and pGADT7-T served as a positive or negative control, respectively. (b) Bimolecular fluorescence complementation (BiFC) assay. Different combinations of plasmid (pUC-SPYNE-MdERF100 and pUC-SPYCE-MdbHLH92; pUC-SPYCE-MdERF100 and pUC-SPYNE-MdbHLH92; pUC-SPYCE-MdERF100 and pUC-SPYNE; pUC-SPYCE and pUC-SPYNE-MdbHLH92; pUC-SPYCE and pUC-SPYNE) were co-transformed in Arabidopsis protoplasts. Yellow fluorescent protein (YFP) signals were detected after $22 \mathrm{~h}$. Scale bar $=10 \mu \mathrm{m}$.

\subsection{Isolation of MdbHLH92 and Its Response to Powdery Mildew}

We performed qRT-PCR analysis to determine whether the expression of MdbHLH92 cloned from the apple cultivar Gala was affected by powdery mildew infection. Compared with the mock control, the expression level of MdbHLH92 was significantly upregulated at 48,72 and $96 \mathrm{hpi}$, reaching a peak at $48 \mathrm{hpi}$ (Figure 6a). The MdbHLH92 gene, located 
on chromosome 16, has a CDS of $708 \mathrm{bp}$, and the MdbHLH92 protein harbors a 49 aa HLH domain (Figure 6b). The molecular weight and theoretical pI of MdbHLH92 are $\sim 27.4 \mathrm{kDa}$ and 9.28, respectively. Furthermore, MdbHLH92 is the most closely related to PbbHLH92-like (Figure 6c). Additionally, MdbHLH92 localized to the cytomembrane (Figure 6d) and showed no transcriptional activation ability in yeast cells (Figure 6e).

(a)

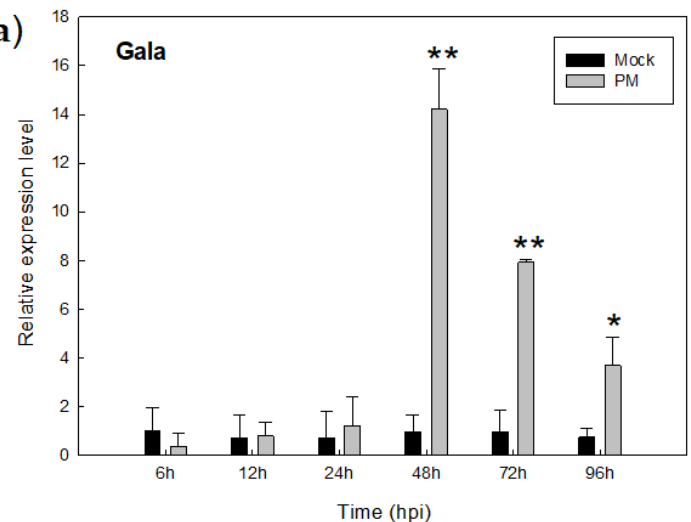

(c)

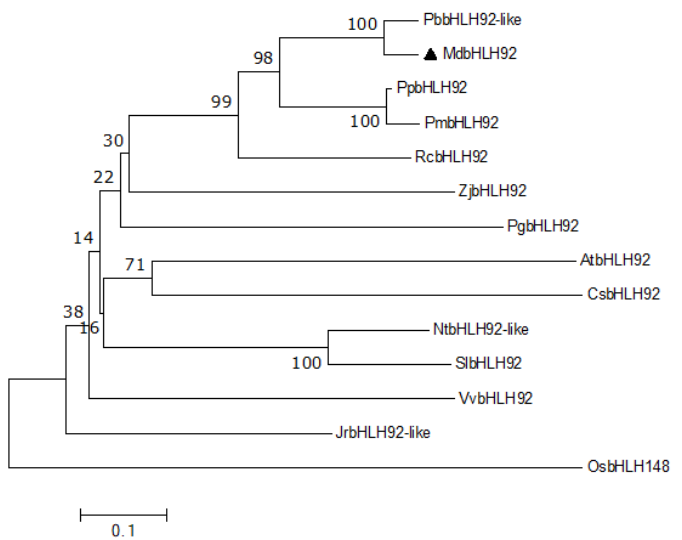

(b)

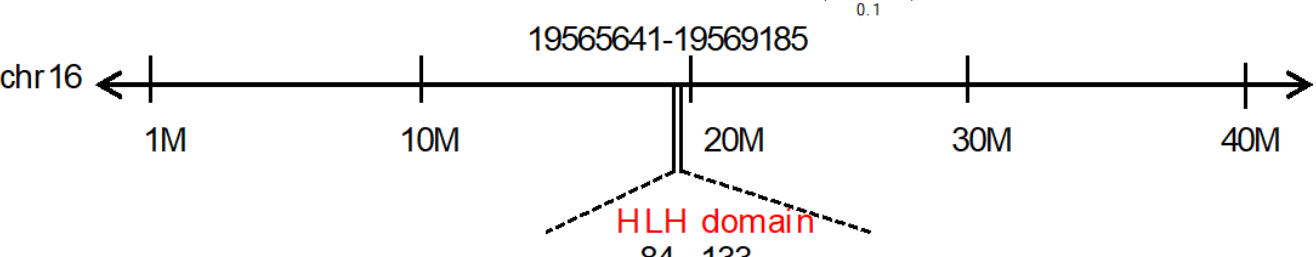

(d) 84133

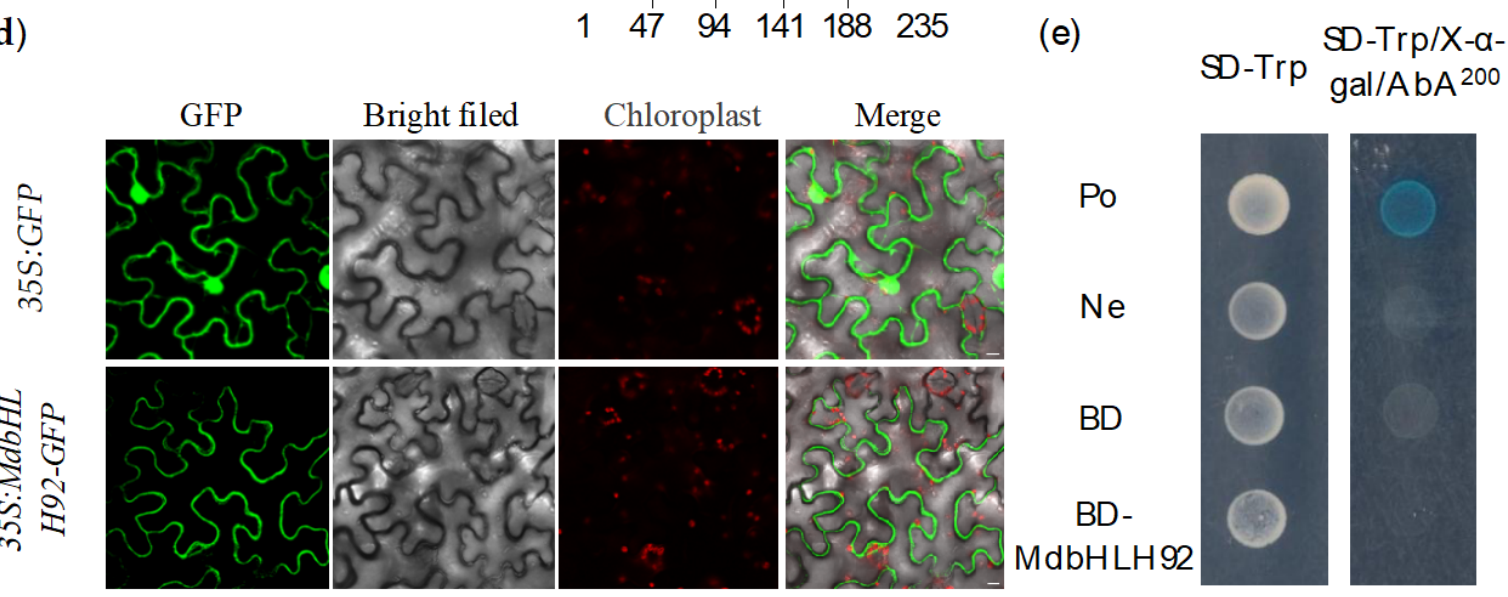

Figure 6. Characterization of MdbHLH92 isolated from the apple cultivar Gala. (a) Expression level of MdbHLH92 in plants infected with powdery mildew. The $x$-axis indicates the time of sampling after inoculation, and the $y$-axis shows the relative gene expression level. Asterisks indicate statistical significance $\left({ }^{*} p<0.05,{ }^{* *} p<0.01\right.$; Student's $t$-test). Error bars were calculated from three biological experiments, and show the standard deviation of the mean. (b) Chromosomal location of MdbHLH92 in the apple genome. (c) Phylogenetic analysis of MdbHLH92 and its homologs. The accession numbers of various proteins are as follows: JrbHLH92-like (XP_018813391.2); PbbHLH92-like (XP_009349402.1); AtbHLH92 (OAO93461.1); VvbHLH92 (XP_002284231.1); OsbHLH148 (XP_015631231.1); PpbHLH92 (XP_007227265.1); NtbHLH92-like (XP_016462348.1); PmbHLH92 (XP_016647633.1); PgbHLH92 (XP_031377233.1); ZjbHLH92 (XP_015887823.1); RcbHLH92 (XP_024194728.1); CsbHLH92 (XP_011649165.2); SlbHLH92 (XP_004247648.1). (d) Subcellular localization of MdbHLH92. GFP signals were observed in Nicotiana benthamiana plants. The pCAMBIA2300-GFP empty vector was used as a control. Scale bar $=10 \mu \mathrm{m}$. (e) Transcriptional activation analysis of MdbHLH92 in yeast. The co-transformation of pGBKT7-53 with pGADT7-T and that of pGBKT7-Lam with pGADT7-T into yeast cells were used as positive and negative controls, respectively. 


\section{Discussion}

Apple is a popular and economically important fruit, but apple trees often encounter various biotic stresses, such as powdery mildew infection, during their life time. According to previous studies, ERF transcription factors respond to various abiotic stresses, although their role in defense against biotic stresses should be given more importance. For instance, the AP2/ERF transcription factor TINY not only promotes drought response, but also suppresses brassinosteroid (BR)-regulated growth in Arabidopsis [29]. In rice (Oryza sativa), both drought tolerance and ethylene emission were reduced in OsERF3-overexpressing transgenic plants compared with the control with inhibited expression of OsERF3, indicating that OsERF3 plays an important role in regulating ethylene biosynthesis and stress response [30]. Studies have shown that MdERF38 plays key roles in inducing anthocyanin biosynthesis under drought stress [31], while MdERF17 is involved in iron deficiency response [32]. However, the disease resistance function of ERF transcription factors in apple need to be explored.

In this study, qRT-PCR analysis showed that, after being inoculated with Podosphaera leucotricha, the expression of MdERF100 was significantly higher at 6, 12 and $72 \mathrm{hpi}$, but lower at 24 and 48 hpi compared with the control group inoculated with sterile water (Figure 1a). The expression level of MdERF100 in Gala plants changed significantly after inoculation with the powdery mildew pathogen, indicating a close relationship between MdERF100 and the powdery mildew resistance mechanism. These results suggest that the MdERF100 confers apple plants with the ability to respond to powdery mildew infection. Furthermore, MdERF100 expression was significantly upregulated after SA, JA, and Eth hormone treatments, indicating that MdERF100 participates in these hormone signaling pathways. Amino acid sequence analyses showed that MdERF100 contains a putative AP2 domain. In soybean (Glycine max), GmERF75 has been shown to localize to the nucleus [33]. Similarly, in Catharanthus roseus, CrERF5 localizes to the nucleus and exhibits transcriptional activation activity [34]. These studies suggest that ERF function as transcriptional activators. In this study, although MdERF100 localized to the nucleus, it did not show transcriptional self-activation in yeast cells. It is possible that MdERF100 requires a post-translational modification or assistance from other proteins to regulate the expression of downstream genes.

In studies of MdERF11 it was shown to enhance resistance to apple ring rot (Botryosphaeria dothidea) via SA signaling pathway [35], and in those of MdERF3 it was shown to respond to Botrytis cinerea [36], suggesting that ERFs in apple might enhance defense responses against fungal diseases. Moreover, six ERF genes (VpERF1, VpERF2, VpERF3, VqERF112, VqERF114 and $V q E R F 72$ ) from Vitis pseudoreticulata and Vitis quinquangularis showed responses to powdery mildew in different expression patterns $[37,38]$. These reports showed ERFs have an important function in regulating the defensive response to powdery mildew. In the current study, three transgenic Arabidopsis lines overexpressing MdERF100 showed an enhanced defense response to powdery mildew compared with WT plants. Additionally, the transgenic lines showed a lower conidiophore count than WT plants at 7 days post-inoculation (dpi). A higher number of dead cells and increased ROS accumulation in transgenic lines suggest that MdERF100 acts as a positive regulator of powdery mildew resistance. The result is consistent with previous studies and makes our findings related to MdERF100 more reliable. Furthermore, hormonal signaling pathways also play an important role in the regulation of disease resistance mechanisms in plants, and the cross-communication between these pathways is antagonistic or synergistic [22,39]. For example, AtERF014 acts as a positive regulator of the SA signaling pathway and a negative regulator of the JA signaling pathway in Arabidopsis plants inoculated with PstDC3000 [40]. Previous researchers investigated the gene participating pathways by measuring the expression of marker genes in hormonal signaling pathways $[35,41]$. The overexpression of MhNPR1 in Nicotiana benthamiana improved the expression of $P R$ genes related to the SA signaling pathway, and could enhance broad-spectrum resistance to apple cultivar pathogens [41]. Moreover, the overexpression of VdGATA2 in Arabidopsis can significantly improve the resistance to powdery mildew through 
increasing the expression of the key genes in the SA signaling pathway (PR1), which indicates that the SA signaling pathway is significant in powdery mildew resistance [42]. In our study, qRT-PCR analysis showed that the expression of a key SA signaling gene (PR1) was high, while that of a key gene involved in JA signaling (PDF1.2) was low (Figure 7). Taken together, these data support the hypothesis that MdERF100 regulates powdery mildew resistance by activating SA and suppressing JA signaling pathways.

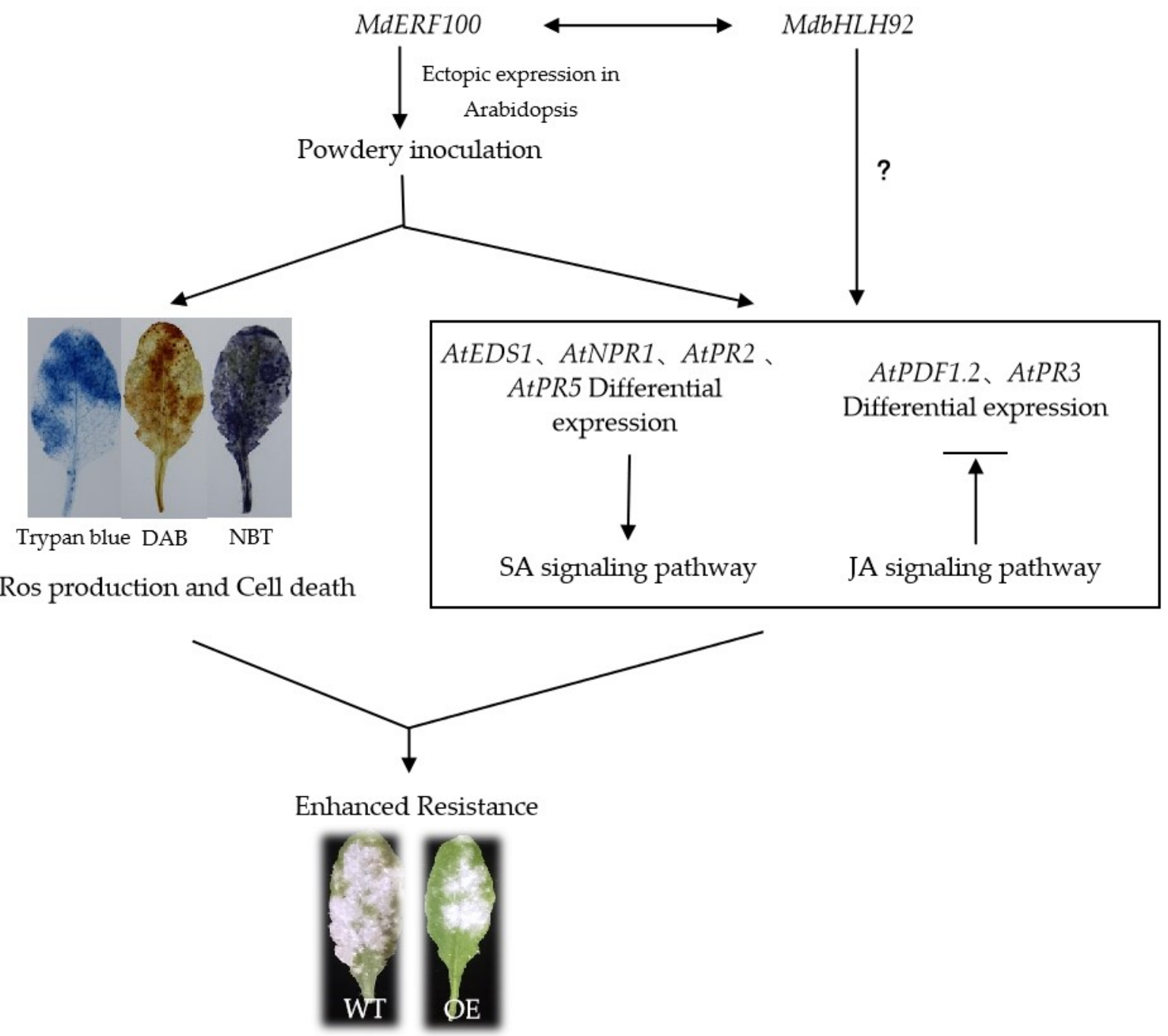

Figure 7. Hypothetical model depicting how MdERF100 functions as a positive regulator of responses to powdery mildew infection.

Previous studies showed that the bHLH transcription factors regulate plant defense against a variety of abiotic and biotic stresses by binding to the E-box cis-acting element in the promoters of stress-responsive genes, thus controlling their expression [43]. In this study, the interaction of MdbHLH92, isolated from Gala, with MdERF100 was confirmed by $\mathrm{Y} 2 \mathrm{H}$ and BiFC assays, suggesting that MdbHLH92 most likely participates in powdery mildew resistance (Figure 7). Whereas MdbHLH92 localized to the cytomembrane, AtbHLH92 localizes to the nucleus [44]. Moreover, we demonstrated the interaction between MdERF100 and MdbHLH92 in the nucleus of Arabidopsis protoplast, which implies that protein activation and migration possibly occurred before the interaction. Furthermore, the expression of MdbHLH92 was significantly upregulated at $48 \mathrm{hpi}$, indicating that MdbHLH92 responds to powdery mildew infection. Further investigations should be carried out to verify the function of MdbHLH92 in powdery mildew resistance through 
heterologous expression analysis in Arabidopsis and homologous expression assays in apple. Moreover, two bHLH transcription factors (TSAR1 and TSAR2) were reported to regulate the biosynthesis of triterpene saponins, which is confirmed to respond to a variety of fungal pathogens [45]. Overexpressing GhbHLH171 in cotton improves its tolerance to the fungus Verticillium dahliae [46]. Although some research provides evidence of the defense function of bHLHs against fungal diseases, our understanding is limited. Previous studies have showed that some bHLH proteins, such as bHLH3 (AT4G16430), bHLH13 (AT1G01260), bHLH14 (AT4G00870) and bHLH17 (AT2G46510/AtAIB), function as negative regulators of JA signaling [46,47], whereas others, such as OsHLH61, act as positive regulators of SA signaling [17]. Coincidentally, our results showed that MdERF100 functions as a positive SA signaling regulator and negative JA signaling regulator, which is supported by the results of our expression analysis, thus providing new insights into the molecular mechanisms underlying the regulation of powdery mildew defense response in apple (Figure 7).

Overall, we showed that MdERF100 acts as a transcription factor and plays a positive regulatory role in enhancing powdery mildew resistance through the SA and JA signaling pathways. Furthermore, we showed that MdERF100 and MdbHLH92 directly interact with each other, which provides a new angle for investigating the transcriptional networks regulated by MdERF100 in response to apple powdery mildew.

\section{Materials and Methods}

\subsection{Plant Materials and Treatments}

Apple (Malus $\times$ domestica cv. Gala) trees were maintained under natural environmental conditions at the White Water Apple Test Station of Northwest A\&F University, Shaanxi, China. Young leaves still attached to the tree were inoculated with a culture of the laboratory strain of Podosphaera leucotricha containing $1 \times 10^{6}$ spores $/ \mathrm{mL}$. Leaves of potted Gala plants grown in the greenhouse were treated with $5 \mathrm{mM} \mathrm{SA}, 50 \mu \mathrm{M}$ methyl jasmonate (MeJA) and $5 \mathrm{mM}$ Eth [48]. Samples were collected at 0, 3, 6, 12 and $24 \mathrm{~h}$ after the hormone treatments. Leaves sprayed with sterile water were used as a control. Arabidopsis thaliana ecotype Columbia (Col-0; WT) plants were grown in a culture chamber under a $16 \mathrm{~h}$ light $/ 8 \mathrm{~h}$ dark photoperiod and $70 \%$ relative humidity. Six- to eight-week-old Arabidopsis plants were subjected to Agrobacterium-mediated transformation to generate stable transgenic lines. Transient transformation of Nicotiana benthamiana (line NC89) plants was performed by agroinfiltration.

\subsection{Bioinformatics Analysis}

The chromosomal location of MdERF100 and MdbHLH92 was predicted by Blat-Search in the Apple Genome Browser. Their conserved protein domains were identified using the Simple Modular Architecture Research Tool (SMART; http: / / smart.embl-heidelberg. de/, accessed on 10 September 2020). The PROSITE database (http: / www.expasy.org / tools / , accessed on 10 September 2020) was used to characterize the physical and chemical properties of MdERF100 and MdbHLH92, and the ProtParam program (http: / web.expasy. $\mathrm{org}$ / protparam/, accessed on 10 September 2020) was used to predict molecular mass. Ten homologous genes of MdERF100 and thirteen of MdbHLH92 were downloaded from NCBI (https://www.ncbi.nlm.nih.gov, accessed on 20 September 2020). The multiple sequence alignment of MdERF100 was generated using the DNAMAN software. The MEGA 7.0 software [49] was used to build a phylogenetic tree of the two genes and their homologous genes with the Neighbor Joining (NJ) method and 1000 bootstrap replicates, respectively.

\subsection{Subcellular Localization and Transcriptional Activation Assays}

The CDSs of MdERF100 and MdbHLH92 were amplified from Gala plants using primers designed by Primer Premier 5.0 (Table S2) in a system containing $100 \mathrm{ng}$ each of the forward and reverse primers, $500 \mathrm{ng}$ of cDNA template and $1 \mathrm{U}$ of PCR Master Mix. The amplified fragment was inserted into the pCAMBIA2300-GFP vector, a reconstructed vector from pCAMBIA2300 (Cambia, Brisbane, QLD, Australia), and the resulting constructs 
(MdERF100-GFP and MdbHLH92-GFP) were introduced into Agrobacterium tumefaciens strain GV3101. Nicotiana benthamiana plants were agroinfiltrated and grown at $22{ }^{\circ} \mathrm{C}$ under $60 \%$ relative humidity and a $16 \mathrm{~h}$ light $/ 8 \mathrm{~h}$ dark photoperiod. GFP signals were observed with a confocal microscope (Olympus FV1000, Tokyo, Japan). The CDSs of MdERF100 and MdbHLH92 were cloned into the pGBKT7 vector, which contains the DNAbinding region of GAL4, using EcoRI and BamHI restriction endonucleases, as described previously [50]. The recombinant vector was transformed into yeast strain Y2H Gold using the Matchmaker ${ }^{\circledR}$ Gold Yeast Two Hybrid System (Takara, Tokyo, Japan), according to the manufacturer's instructions. The co-transformations of pGBKT7-53 with pGADT7-T and of pGBKT7-Lam with pGADT7-T into yeast cells were used as positive and negative controls, respectively. The transformants were grown at $30^{\circ} \mathrm{C}$ for 3 days. Transcriptional activation was determined by blue colonies on selective medium (SD-Trp) supplemented with $40 \mu \mathrm{g} / \mathrm{mL} \mathrm{X- \alpha -Gal} \mathrm{and} 200 \mathrm{ng} / \mathrm{mL}$ AbA. The primers used for plasmid construction are listed in Table S2.

\subsection{Inoculation of Arabidopsis Plants with Powdery Mildew Pathogen}

The MdERF100 CDS was cloned downstream of the CaMV 35S promoter in the plant expression vector PCAMBIA2300-GFP, and the resultant construct was introduced into A. tumefaciens strain GV3101. The floral dip method [51] was used to transform Arabidopsis seedlings. Seeds of T0 plants were selected on Murashige and Skoog (MS) medium supplemented with $50 \mathrm{mg} / \mathrm{L}$ kanamycin. Homozygous T3 lines were selected from three independent (T1) lines, and the homozygous T3 plants were used for subsequent experiments. T3 plants with five to seven leaves were inoculated with Golovinomyces cichoracearum UCSC1, an obligate parasitic powdery mildew pathogen of Arabidopsis stored on the leaves of WT plant, through rubbing the healthy leaves with infected leaves gently. WT plants were used as the control. Samples were collected before inoculation $(0 \mathrm{~h})$ and at 24,72 and $120 \mathrm{hpi}$. All samples were immediately frozen in liquid nitrogen and stored at $-80^{\circ} \mathrm{C}$. To perform trypan blue, DAB, and NBT staining, samples were collected at $168 \mathrm{hpi}$ (7 dpi).

\subsection{RNA Isolation and Gene Expression Analysis}

Total RNA was extracted from using the E.Z.N.A. Plant RNA Kit (R6827-01, Omega Bio-tek, Doraville, GA, USA). The reverse transcription reaction was performed in two steps with the Evo M-MLV RT Kit with gDNA Clean for qPCRII (AG11711, Accurate Biotechnology, Changsha, Hunan, China), referring to the manufacturer's instructions. Then, the synthesized cDNA was diluted six-fold to use as a template in qRT-PCR, which was performed using ChamQTM SYBR ${ }^{\circledR}$ qPCR Master Mix (Vazyme) for fluorescence quantitation in a reaction system of $20 \mu \mathrm{L}$ (Table S1) under the following conditions: $95^{\circ} \mathrm{C}$ for $30 \mathrm{~s}$, followed by 42 cycles at $95^{\circ} \mathrm{C}$ for $10 \mathrm{~s}$ and $60^{\circ} \mathrm{C}$ for $30 \mathrm{~s}$. Relative expression levels were analyzed with the $2^{-\Delta \Delta C T}$ method. Graphs were generated with SigmaPlot 12.0 (Systat Software, San Jose, CA, USA) using relative expression values.

\section{6. $\mathrm{Y} 2 \mathrm{H}$ and BiFC Assays}

Ten proteins were predicted to interact with MdERF100 using a Search Tool for the Retrieval of Interacting Genes/Proteins (STRING; https: / / string-db.org/cgi/input, accessed on 25 May 2020). After confirming the CDSs of genes encoding these proteins with the NCBI database (http:/ / blast.ncbi.nlm.nih.gov/Blast.cgi, accessed on 25 May 2020), gene-specific primers containing EcoRI and BamHI restriction sites were designed with Primer Premier 5.0 (Table S2). Then, the CDSs of five genes (MdCSC1, MdBIP, MdbHLH92, $M d P A E 1$ and $M d U B Q$ ) were cloned successfully from Gala and inserted into the pGADT7 vector. The resulting vectors were co-transformed into the yeast strain $\mathrm{Y} 2 \mathrm{H}$ and cultivated on SD/-Trp/-Leu (DDO), SD/-Ade-His-Leu-Trp (QDO) and QDO supplemented with $40 \mu \mathrm{g} / \mathrm{mL} \mathrm{X- \alpha -Gal} \mathrm{and} 200 \mathrm{ng} / \mathrm{mL} \mathrm{AbA}(\mathrm{QDO} / \mathrm{X} / \mathrm{A})$. The growth of yeast colonies was observed after 3-4 days; blue colonies on QDO/X/A indicated protein-protein interaction. 
Full-length CDSs of MdERF100 and MdbHLH92 were cloned into pUCSPY-CE and pUCSPYNE vectors [52] using XhoI and KpnI restriction enzymes. The recombinant plasmids were co-transformed into Arabidopsis protoplasts as described previously [53]. After $22 \mathrm{~h}$, yellow fluorescent protein (YFP) signals were visualized by confocal laser scanning microscope (LEICA TCS SP8, Leica, Bensheim, Germany). The primers used for plasmid construction are listed in Table S2.

\section{Conclusions}

Consistent with its presumed identity as a transcriptional regulator, MdERF100 localized to the nucleus and did not activate gene transcription. The overexpression of MdERF100 in Arabidopsis enhanced resistance to powdery mildew and influenced the expression of genes involved in SA and JA signaling pathways. Additionally, MdERF100 showed direct physical interaction with MdbHLH92 in $\mathrm{Y} 2 \mathrm{H}$ and BiFC assays. Thus, this study provides preliminary information about the function of MdERF100 in powdery mildew resistance.

Supplementary Materials: The following are available online at https://www.mdpi.com/article/10 $.3390 /$ ijms22115713/s1.

Author Contributions: Conceptualization, Z.Z., X.W. and H.G.; methodology, X.W. and H.G.; formal analysis, Y.Z. (Yiping Zhang), L.Z.; investigation, Y.Z. (Yiping Zhang), L.Z., H.M., Y.Z. (Yichu Zhang), X.Z. and M.J.; resources, X.W. and H.G.; writing - original draft preparation, Y.Z. (Yiping Zhang) and L.Z.; writing—review and editing, Y.Z. (Yiping Zhang), L.Z., X.Z., S.v.N. and B.A.; funding acquisition, H.G. All authors have read and agreed to the published version of the manuscript.

Funding: The work was supported by the Shaanxi Provincial Key Research and Development (R\&D) Program (2020zdzx03-06-02-02) and the China Agriculture Research System (CARS-27).

Institutional Review Board Statement: Not applicable.

Informed Consent Statement: Not applicable.

Data Availability Statement: The data supporting the findings of this study are available within the article and its Supplementary Materials.

Acknowledgments: The authors thank all editors and reviewers for their comments on this manuscript.

Conflicts of Interest: The authors declare no conflict of interest.

\section{References}

1. Tian, X.; Zhang, L.; Feng, S.; Zhao, Z.; Wang, X.; Gao, H. Transcriptome Analysis of Apple Leaves in Response to Powdery Mildew (Podosphaera leucotricha) Infection. Int. J. Mol. Sci. 2019, 20, 2326. [CrossRef]

2. Gañán, L.; White, I.R.; Friesen, M.; Peever, T.; Amiri, A. A Genome Resource for the Apple Powdery Mildew Pathogen Podo-sphaera leucotricha. Phytopathology 2020, 110, 1756-1758. [CrossRef]

3. Turechek, W.W.; Carroll, J.E.; Rosenberger, D.A. Powdery Mildew of Apple. 2005. Available online: https:/ / ecommons.cornell. edu/handle/1813/43120 (accessed on 10 May 2019).

4. Pessina, S.; Angeli, D.; Martens, S.; Visser, R.G.; Bai, Y.; Salamini, F.; Velasco, R.; Schouten, H.J.; Malnoy, M. The knock-down of the expression of MdMLO19 reduces susceptibility to powdery mildew (Podosphaera leucotricha) in apple (Malus domestica). Plant Biotechnol. J. 2016, 14, 2033-2044. [CrossRef] [PubMed]

5. Li, M.-Y.; Xu, Z.-S.; Huang, Y.; Tian, C.; Wang, F.; Xiong, A.-S. Genome-wide analysis of AP2/ERF transcription factors in carrot (Daucus carota L.) reveals evolution and expression profiles under abiotic stress. Mol. Genet. Genom. 2015, 290, $2049-2061$. [CrossRef]

6. Wang, L. Studies on transcription factor ERF regulating disease resistance-related genes in Chinese wild Vitis quinquangularis. Ph.D. Thesis, Northwest A\&F University, Xianyang, China, 2019.

7. Gutterson, N.; Reuber, T.L. Regulation of disease resistance pathways by AP2/ERF transcription factors. Curr. Opin. Plant Biol. 2004, 7, 465-471. [CrossRef]

8. Liu, M.; Ma, F.; Wu, F.; Jiang, C.; Wang, Y. Expression of stilbene synthase VqSTS6 from wild Chinese Vitis quinquangularis in grapevine enhances resveratrol production and powdery mildew resistance. Planta 2019, 250, 1997-2007. [CrossRef]

9. Zhou, Z.; Wang, J.; Zhang, S.; Yu, Q.; Lan, H. Investigation of the Nature of CgCDPK and CgbHLH001 Interaction and the Function of bHLH Transcription Factor in Stress Tolerance in Chenopodium glaucum. Front. Plant Sci. 2021, 11, 603298. [CrossRef] 
10. Toledo-Ortiz, G.; Huq, E.; Quail, P.H. The Arabidopsis Basic/Helix-Loop-Helix Transcription Factor Family. Plant Cell 2003, 15, 1749-1770. [CrossRef] [PubMed]

11. Feller, A.; Machemer, K.; Braun, E.L.; Grotewold, E. Evolutionary and comparative analysis of MYB and bHLH plant transcription factors. Plant J. 2011, 66, 94-116. [CrossRef] [PubMed]

12. Shen, T.; Wen, X.; Wen, Z.; Qiu, Z.; Hou, Q.; Li, Z.; Mei, L.; Yu, H.; Qiao, G. Genome-wide identification and expression analysis of bHLH transcription factor family in response to cold stress in sweet cherry (Prunus avium L.). Sci. Hortic. 2021, $279,109905$. [CrossRef]

13. Qiu, J.-R.; Huang, Z.; Xiang, X.-Y.; Xu, W.-X.; Wang, J.-T.; Chen, J.; Song, L.; Xiao, Y.; Li, X.; Ma, J.; et al. MfbHLH38, a Myrothamnus flabellifolia bHLH transcription factor, confers tolerance to drought and salinity stresses in Arabidopsis. BMC Plant Biol. 2020, 20, 1-14. [CrossRef]

14. Rajappa, S.; Krishnamurthy, P.; Kumar, P.P. Regulation of AtKUP2 Expression by bHLH and WRKY Transcription Factors Helps to Confer Increased Salt Tolerance to Arabidopsis thaliana Plants. Front. Plant Sci. 2020, 11, 1311. [CrossRef]

15. Lockhart, J. Personal Trainer: bHLH121 functions upstream of a transcriptional network of heavy lifters involved in bal-ancing iron levels. Plant Cell 2020, 32, 293-294. [CrossRef] [PubMed]

16. Kim, J.-G.; Mudgett, M.B. Tomato bHLH132 Transcription Factor Controls Growth and Defense and Is Activated by Xanthomonas euvesicatoria Effector XopD During Pathogenesis. Mol. Plant-Microbe Interact. 2019, 32, 1614-1622. [CrossRef]

17. Wang, M.; Yang, D.; Ma, F.; Zhu, M.; Shi, Z.; Miao, X. OsHLH61-OsbHLH96 influences rice defense to brown planthopper through regulating the pathogen-related genes. Rice 2019, 12, 9. [CrossRef]

18. Ke, Y.; Kang, Y.; Wu, M.; Liu, H.; Hui, S.; Zhang, Q.; Li, X.; Xiao, J.; Wang, S. Jasmonic Acid-Involved OsEDS1 Signaling in Rice-Bacteria Interactions. Rice 2019, 12, 1-12. [CrossRef]

19. Matthews, B.F.; Beard, H.; Brewer, E.; Kabir, S.; MacDonald, M.H.; Youssef, R.M. Arabidopsis genes, AtNPR1, AtTGA2 and AtPR-5, confer partial resistance to soybean cyst nematode (Heterodera glycines) when overexpressed in transgenic soy-bean roots. BMC Plant Biol. 2014, 14, 96. [CrossRef] [PubMed]

20. Yuan, P.; Jewell, J.B.; Behera, S.; Tanaka, K.; Poovaiah, B.W. Distinct Molecular Pattern-Induced Calcium Signatures Lead to Different Downstream Transcriptional Regulations via AtSR1/CAMTA3. Int. J. Mol. Sci. 2020, 21, 8163. [CrossRef]

21. Wu, Y.; Zhang, D.; Chu, J.; Boyle, P.; Wang, Y.; Brindle, I.D.; De Luca, V.; Després, C. The Arabidopsis NPR1 protein is a re-ceptor for the plant defense hormone salicylic acid. Cell Rep. 2012, 1, 639-647. [CrossRef] [PubMed]

22. Mur, L.A.; Kenton, P.; Atzorn, R.; Miersch, O.; Wasternack, C. The Outcomes of Concentration-Specific Interactions between Salicylate and Jasmonate Signaling Include Synergy, Antagonism, and Oxidative Stress Leading to Cell Death. Plant Physiol. 2006, 140, 249-262. [CrossRef]

23. Jayakannan, M.; Bose, J.; Babourina, O.; Rengel, Z.; Shabala, S. Salicylic acid in plant salinity stress signalling and tolerance. Plant Growth Regul. 2015, 76, 25-40. [CrossRef]

24. Zhang, X.; Ménard, R.; Li, Y.; Coruzzi, G.M.; Heitz, T.; Shen, W.-H.; Berr, A. Arabidopsis SDG8 Potentiates the Sustainable Transcriptional Induction of the Pathogenesis-Related Genes PR1 and PR2 During Plant Defense Response. Front. Plant Sci. 2020, 11, 277. [CrossRef]

25. Colavolpe, M.B.; Villarreal, N.M.; Langer, S.E.; Romero, F.M.; Martínez, G.A.; Saini, A.; Ruiz, O.A.; Marina, M. Burkholderia sp. Strain AU4i Promotes Arabidopsis Growth and Increases Plant Defence Response to Necrotrophic Pathogens. J. Plant Growth Regul. 2020, 1-11. [CrossRef]

26. Yu, Y.; Bian, L.; Wan, Y.; Jiao, Z.; Yu, K.; Zhang, G.; Guo, D. Grape (Vitis vinifera) VvDOF3 functions as a transcription activator and enhances powdery mildew resistance. Plant Physiol. Bioch. 2019, 143, 183-189. [CrossRef] [PubMed]

27. Xing, L.; Di, Z.; Yang, W.; Liu, J.; Li, M.; Wang, X.; Cui, C.; Wang, X.; Wang, X.; Zhang, R.; et al. Overexpression of ERF1-V from Haynaldia villosa Can Enhance the Resistance of Wheat to Powdery Mildew and Increase the Tolerance to Salt and Drought Stresses. Front. Plant Sci. 2017, 8, 1948. [CrossRef] [PubMed]

28. Chen, X.-K.; Zhang, J.-Y.; Zhang, Z.; Du, X.-L.; Du, B.-B.; Qu, S.-C. Overexpressing MhNPR1 in transgenic Fuji apples enhances resistance to apple powdery mildew. Mol. Biol. Rep. 2012, 39, 8083-8089. [CrossRef]

29. Xie, Z.; Nolan, T.; Jiang, H.; Tang, B.; Zhang, M.; Li, Z.; Yin, Y. The AP2/ERF transcription factor TINY modulates brassinosteroid-regulated plant growth and drought responses in Arabidopsis. Plant Cell 2019, 31, 1788-1806. [CrossRef]

30. Zhang, H.; Zhang, J.; Quan, R.; Pan, X.; Wan, L.; Huang, R. EAR motif mutation of rice OsERF3 alters the regulation of eth-ylene biosynthesis and drought tolerance. Planta 2013, 237, 1443-1451. [CrossRef]

31. An, J.; Zhang, X.; Bi, S.; You, C.; Wang, X.; Hao, Y. The ERF transcription factor MdERF38 promotes drought stress-induced anthocyanin biosynthesis in apple. Plant J. 2019, 101, 573-589. [CrossRef]

32. Cheng, L.; Zhao, T.; Wu, Y.; Wang, H.; Zhang, Z.; Zhang, D.; Wang, S.; Wang, Y. Identification of AP2/ERF genes in apple (Malus $\times$ domestica) and demonstration that MdERF017 enhances iron deficiency tolerance. Plant Cell Tiss. Org. 2020, 143, 465-482. [CrossRef]

33. Zhao, M.-J.; Yin, L.-J.; Liu, Y.; Ma, J.; Zheng, J.-C.; Lan, J.-H.; Fu, J.-D.; Chen, M.; Xu, Z.-S.; Ma, Y.-Z. The ABA-induced soybean ERF transcription factor gene GmERF75 plays a role in enhancing osmotic stress tolerance in Arabidopsis and soybean. BMC Plant Biol. 2019, 19, 1-14. [CrossRef] 
34. Pan, Q.; Wang, C.; Xiong, Z.; Wang, H.; Fu, X.; Shen, Q.; Peng, B.; Ma, Y.; Sun, X.; Tang, K. CrERF5, an AP2/ERF Transcription Factor, Positively Regulates the Biosynthesis of Bisindole Alkaloids and Their Precursors in Catharanthus roseus. Front. Plant Sci. 2019, 10, 931. [CrossRef] [PubMed]

35. Wang, J.-H.; Gu, K.-D.; Han, P.-L.; Yu, J.-Q.; Wang, C.-K.; Zhang, Q.-Y.; You, C.-X.; Hu, D.-G.; Hao, Y.-J. Apple ethylene response factor MdERF11 confers resistance to fungal pathogen Botryosphaeria dothidea. Plant Sci. 2020, 291, 110351. [CrossRef]

36. Akagi, A.; Dandekar, A.M.; Stotz, H.U. Resistance of Malus domestica fruit to Botrytis cinerea depends on endogenous eth-ylene biosynthesis. Phytopathology 2011, 101, 1311-1321. [CrossRef]

37. Zhu, Z.; Shi, J.; Xu, W.; Li, H.; He, M.; Xu, Y.; Xu, T.; Yang, Y.; Cao, J.; Wang, Y. Three ERF transcription factors from Chinese wild grapevine Vitis pseudoreticulata participate in different biotic and abiotic stress-responsive pathways. J. Plant Physiol. 2013, 170, 923-933. [CrossRef] [PubMed]

38. Wang, L.; Liu, W.; Wang, Y. Heterologous expression of Chinese wild grapevine VqERFs in Arabidopsis thaliana enhance resistance to Pseudomonas syringae pv. tomato DC3000 and to Botrytis cinerea. Plant Sci. 2020, 293, 110421. [CrossRef] [PubMed]

39. Schenk, P.M.; Kazan, K.; Wilson, I.; Anderson, J.P.; Richmond, T.; Somerville, S.C.; Manners, J.M. Coordinated plant defense responses in Arabidopsis revealed by microarray analysis. Proc. Natl. Acad. Sci. USA 2000, 97, 11655-11660. [CrossRef]

40. Zhang, H.; Hong, Y.; Huang, L.; Li, D.; Song, F. Arabidopsis AtERF014 acts as a dual regulator that differentially modulates immunity against Pseudomonas syringae pv. tomato and Botrytis cinerea. Sci. Rep. 2016, 6, 30251. [CrossRef]

41. Zhang, J.; Qiao, Y.; Lv, D.; Gao, Z.; Qu, S.; Zhang, Z. Malus hupehensis NPR1 induces pathogenesis-related protein gene expression in transgenic tobacco. Plant Biol. 2012, 14, 46-56. [CrossRef] [PubMed]

42. Yu, Y.-H.; Bian, L.; Yu, K.-K.; Yang, S.-D.; Zhang, G.-H.; Guo, D.-L. Grape (Vitis davidii) VdGATA2 functions as a transcription activator and enhances powdery mildew resistance via the active oxygen species pathway. Sci. Hortic. 2020, $267,109327$. [CrossRef]

43. Robinson, K.A.; Koepke, J.I.; Kharodawala, M.; Lopes, J.M. A network of yeast basic helix-loop-helix interactions. Nucleic Acids Res. 2000, 28, 4460-4466. [CrossRef] [PubMed]

44. Jiang, Y.; Yang, B.; Deyholos, M.K. Functional characterization of the Arabidopsis bHLH92 transcription factor in abiotic stress. Mol. Genet. Genom. 2009, 282, 503-516. [CrossRef] [PubMed]

45. Meraj, T.A.; Fu, J.; Raza, M.A.; Zhu, C.; Shen, Q.; Xu, D.; Wang, Q. Transcriptional Factors Regulate Plant Stress Responses Through Mediating Secondary Metabolism. Genes 2020, 11, 346. [CrossRef] [PubMed]

46. He, X.; Zhu, L.; Wassan, G.M.; Wang, Y.; Miao, Y.; Shaban, M.; Hu, H.; Sun, H.; Zhang, X. GhJAZ2 attenuates cotton resistance to biotic stresses via the inhibition of the transcriptional activity of GhbHLH171. Mol. Plant Pathol. 2017, 19, 896-908. [CrossRef] [PubMed]

47. Song, S.; Qi, T.; Fan, M.; Zhang, X.; Gao, H.; Huang, H.; Wu, D.; Guo, H.; Xie, D. The bHLH Subgroup IIId Factors Negatively Regulate Jasmonate-Mediated Plant Defense and Development. PLoS Genet. 2013, 9, e1003653. [CrossRef] [PubMed]

48. Lui, S.; Luo, C.; Zhu, L.; Sha, R.; Qu, S.; Cai, B.; Wang, S. Identification and expression analysis of WRKY transcription factor genes in response to fungal pathogen and hormone treatments in apple (Malus domestica). J. Plant Biol. 2017, 60, 215-230. [CrossRef]

49. Kumar, S.; Stecher, G.; Tamur, A.K. MEGA7: Molecular Evolutionary Genetics Analysis version 7.0 for bigger datasets. Mol. Biol. Evol. 2016, 33, 1870-1874. [CrossRef]

50. Zhang, X.; Zhang, L.; Ji, M.; Wu, Y.; Zhang, S.; Zhu, Y.; Yao, J.; Li, Z.; Gao, H.; Wang, X. Genome-wide identification and ex-pression analysis of the B-box transcription factor gene family in grapevine. BMC Genom. 2021, 22, 221. [CrossRef]

51. Clough, S.J.; Bent, A.F. Floral dip: A simplified method for Agrobacterium-mediated transformation of Arabidopsis thaliana. Plant J. 1998, 16, 735-743. [CrossRef]

52. Walter, M.; Chaban, C.; Schütze, K.; Batistic, O.; Weckermann, K.; Näke, C.; Blazevic, D.; Grefen, C.; Schumacher, K.; Oecking, C.; et al. Visualization of protein interactions in living plant cells using bimolecular fluorescence complementa-tion. Plant J. 2004, 40, 428-438. [CrossRef]

53. Yoo, S.-D.; Cho, Y.-H.; Sheen, J. Arabidopsis mesophyll protoplasts: A versatile cell system for transient gene expression analysis. Nat. Protoc. 2007, 2, 1565-1572. [CrossRef] [PubMed] 